\title{
Surgical treatment of distal femur fractures using locking compression plate
}

\author{
Tushar Agarwal ${ }^{1, *}$, Kumar Saurav ${ }^{2}$, Siddharth Jadhav ${ }^{3}$, Ankur Kumar ${ }^{4}$, Manoj Pudari ${ }^{5}$ \\ ${ }^{1}$ Professor, ${ }^{2-5}$ Resident, Dept. of Orthopaedics, Dr. D.Y. Patil Medical College, Pune, Maharashtra, India
}

*Corresponding Author:

Email: tushar.agarwal@rediffmail.com

\begin{abstract}
Distal femur fractures have been a challenge to the orthopaedic fraternity for long time. Improvement in implant material and design are expected to fetch better results in due course of time. Advent and use of locking compression plate has shown good results but requires more corroborative evidence to advocate its uses in different morphology of distal femur fracture. The present study was undertaken to evaluate clinical, radiological union and the complications associated with locking compression plate.
\end{abstract}

Keywords: Distal femur fracture, Locking compression plate.

\section{Introduction}

Distal end femur fractures are not new to the orthopedic fraternity. It has been distinguished as an orthopedic issue all through history. These fractures constitute 6 to $7 \%$ of all femur fractures. ${ }^{1}$ Bimodal distribution is noted on the basis of age and gender. High-energy distal femur fractures happen mostly in males in the age group of 15 to 50 years and mostly low-energy fractures happen in osteoporotic females aged $>50$ years. The commonest mode of injury due to high-energy trauma is motor vehicle accident (53\%) and domestic fall happens to cause low-energy fractures. (33\%). ${ }^{2}$

Verifiably, these fractures tend to happen in patients with polytrauma and in elderly patients. These fractures are unstable fractures, so they are difficult to treat. Proximity to knee joint renders them vulnerable to limited range of motion. It additionally adds to different complications i.e. infection, fixed flexion deformity, knee stiffness. These complications make treatment of distal femoral fractures a troublesome undertaking for an orthopedic surgeon. ${ }^{1}$

During 1960s, non-operative treatment strategies, for example, traction and cast bracing, delivered better outcomes over operative treatment in light of the absence of sufficient internal fixation devices. ${ }^{3,4}$ With growing popularity of indirect reduction technique for re-establishing alignment of the limb, it has been noted to have increased rate of fracture healing and decreased rate of infection, decreased failure of fixation and reduced requirement of bone graft.

Conservative modalities maybe used for fixation of fractures which are undisplaced or displaced minimally in a patient of old age and with limited functional requirement. Conservative treatment include skeletal traction or splints and mobilisation with partial weight bearing and eventually with cast or functional brace. Xrays are taken weekly to fortnightly in the initial six weeks to confirm whether reduction is maintained or not. Gradually patient is allowed partial to full weight bearing with joint mobilisation keeping in view the $\mathrm{X}$ ray and clinical correlates of fracture union.
Overall, conservative approach has not proven effective for displaced fractures. Butt et $\mathrm{al}^{5}$ assessed operative versus non-operative treatment for displaced distal fractures of femur in the old age group in a RCT. This involved either randomizing the patients to surgical management using dynamic condylar screw $(n=17)$ or giving skeletal traction for 6 to 8 weeks followed by functional bracing $(n=19)$. Good to excellent outcome was noted in 53\% of surgicallymanaged cases while $31 \%$ in conservatively managed cases. The patients who were conservatively managed showed higher chances of developing DVT (regardless of coumadin treatment), UTI and respiratory tract infections, bed sores, non-unions, malunions and pin tract infections. Average stay at hospital in case of conservatively managed cases was 9 weeks. Consequently, Butt et $\mathrm{al}^{5}$ advocated surgical management of displaced distal femur fractures in older age group and conservative management only in cases where patients were unfit for surgery.

Surgical intervention is treatment of choice for displaced and/or open fractures along with the ones having vascular injury. Aim of management incorporate anatomical restoration of the articulating surfaces, restoring lower extremity alignment, early knee range of motion, and early patient mobilization.

Early treatment of these fractures normally incorporates an adequately padded long leg splint to minimize soft tissue compromise. In simple or compound fractures associated with high velocity injury, especially in polytrauma patients, a few specialists advocate use of knee-spanning external fixator until the point when definitive fixation is possible. ${ }^{6-8}$ It helps to re-establish alignment, decreases chances of further soft tissue trauma and

also enhances patient comfort and mobility. Calcaneum or proximal tibia can be used for skeletal traction as other alternatives to stabilize the fracture.

Numerous choices can be availed for final fixation of these fractures and they comprise of external fixators, intramedullary nails, and plate osteosynthesis with open reduction and internal fixation or minimally 
invasive plate osteosynthesis (MIPO).

As orthopedic surgery has developed, patterns noted in fixation of supracondylar and intercondylar femur fractures now often include surgical management. Implants used for fracture fixation are $95^{\circ}$ angled blade plate, dynamic condylar screw plate, condylar buttress plate as well as retrograde supracondylar interlocking nail.

In any case, as the pattern of the fractures has moved on from simple extra-articular to intercondylar and involving metaphysis with comminution, the above mentioned implants have been rendered imperfect for fixation. Locking plate and bicondylar plating strategies have been recommended. ${ }^{10-13}$ But with bicondylar plating, there is an added risk of more soft tissue trauma and periosteal stripping on either sides of the bone, bringing about reduced vascularity and more chances of non-union and implant failure.

The less invasive stabilization system (LISS) provides rigid angle fixation in distal femur as well as minimally invasive fixation of the femur shaft ${ }^{14}$ But stiffness of implant and premature ambulation brings about implant failure. ${ }^{15}$ The decision of treatment is not obvious in light of the fact that number of treatment choices are available in these fractures. Each has its benefits and potential complications. An exhaustive learning of basic principles is fundamental to determine a correct system to be done at perfect time.

Distal femoral locking pressure plate (DF-LCP) has a small applicator with locking and compression screw for fracture fixation. It gives fixed angle amongst plate and screw interface. It dispenses with screw toggle and diminishes motion at fracture site, so periosteal blood supply is preserved. It likewise gives much stronger fixation in osteoporotic bone. ${ }^{16,17}$

However, there have been few results examining distal femur fractures treated with locking compression plate, demonstrating better outcomes when contrasted with the conventional treatment (antegrade nailing or plate and screw fixation). Each existing technique has drawbacks like, non-operative care brings about prolonged hospitalization and immobilization, conventional dynamic condylar screw and condylar buttress plate are inconsistent in osteoporotic bones and the issue of displaced intraarticular fracture isn't settled with ante grade nailing. Likewise more data is required in India to assess its utilization all the more so with increased in motor vehicle accident cases coming to the emergency.

Henceforth this investigation was intended to assess and explore locking compression plate fixation in distal end femur fractures which is expected to furnish a steady fixation with least exposure, early mobilization, less complications and a better quality of life.

\section{Materials and Methods}

The present study was conducted at Dr. D.Y Patil medical college Hospital and Research Centre, Pune during the period of June 2014 to September 2016.

During the period 30 patients of distal femur fracture were treated surgically.

Study Design: The study design was prospective study Study Period and Duration: The present study was conducted from June 2015 to September 2017.

Place: This study was done at the Department of Orthopaedics, Dr. D.Y Patil medical college Hospital and Research Centre, pune

\section{Selection Criteria: Inclusion Criteria:}

1. Participant giving consent for enrollment in the study.

2. All skeletally mature patients (> 18 years).

3. Fracture extending into distal $15 \mathrm{~cm}$ of the femur including extra articular, partial articular (except 33-b2) or complete articular.

Fractures classified using the AO Classification system

Exclusion Criteria:

1. Patients not willing to participate in the study.

2. Skeletally immature patients. ( $<18$ years).

3. Fracture with vascular injury needing repair.

4. Fractures older than three weeks in case of closed fracture or more than 24 hours in open fractures.

5. Pathological fractures.

6. Compound fractures of the lower end femur with extensive soft tissue damage.

7. Old fractures with complications like infection, delayed union, nonunion and malunion.

8. Fractures with associated tibia plateau fracture.

\section{Instruments}

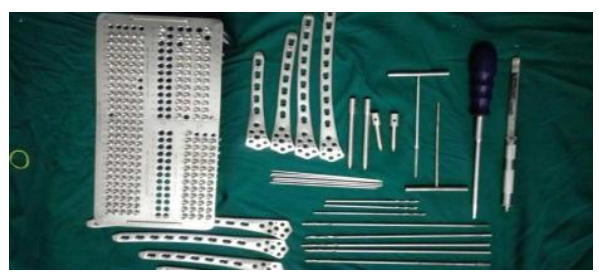

Fig. 1: Distal femur locking compression plate

Locking Screws: $4.9 \mathrm{~mm}$ and $6.5 \mathrm{~mm}$ with drill bits of $4.3 \mathrm{~mm}$ and $4.5 \mathrm{~mm}$ respectively Non-locking screws: $4.5 \mathrm{~mm}$ with drill bit of $3.2 \mathrm{~mm}$

Screw Driver: Hexagonal Depth gauge, power drill.

\section{Observations and Results}

Table 1: Complications-post operative late

\begin{tabular}{|l|c|c|}
\hline Complications & \multicolumn{2}{|c|}{ Distribution $(\mathbf{n}=\mathbf{3 0})$} \\
\cline { 2 - 3 } & Number & Percentage \\
\hline $\begin{array}{l}\text { Chronic } \\
\text { infection }\end{array}$ & 3 & 10.00 \\
\hline Knee stiffness & 3 & 10.00 \\
\hline Malalignment & 3 & 10.00 \\
\hline
\end{tabular}

In the present study, late postoperative complications included chronic infection (10\%), knee stiffness (10\%), malalignment (10\%). 
Table 2: Radiological and clinical union

\begin{tabular}{|l|c|c|c|c|}
\hline \multirow{2}{*}{ Follow up } & \multicolumn{4}{|c|}{ Distribution ( n = 30) } \\
\cline { 2 - 5 } & Number & Percentage & Number & Percentage \\
\hline 3 months & 14 & 46.67 & $\mathbf{1 4}$ & 46.67 \\
\hline 6 months & 14 & 46.67 & $\mathbf{1 4}$ & 46.67 \\
\hline
\end{tabular}

In the present study, the radiological union was noted in $46.67 \%$ patients each at three months and six months. In this study, clinical union was noted in $46.67 \%$ patients each at three months and six months.

Table 3: Association between type of fractures and outcome

\begin{tabular}{|l|c|c|c|c|c|c|}
\hline \multirow{2}{*}{$\begin{array}{l}\text { Type of } \\
\text { fracture }\end{array}$} & \multicolumn{6}{|c|}{ Distribution ( $\mathbf{n}=30)$} \\
\cline { 2 - 7 } & \multicolumn{2}{|c|}{ Excellent } & \multicolumn{2}{|c|}{ Good } & \multicolumn{2}{c|}{ Fair } \\
\cline { 2 - 7 } & No. & $\mathbf{\%}$ & No. & $\mathbf{\%}$ & No. & \% \\
\hline A1 & 5 & 100.00 & 0 & 0.00 & 0 & 0.00 \\
\hline A2 & 3 & 100.00 & 0 & 0.00 & 0 & 0.00 \\
\hline A3 & 3 & 100.00 & 0 & 0.00 & 0 & 0.00 \\
\hline B1 & 2 & 100.00 & 0 & 0.00 & 0 & 0.00 \\
\hline C1 & 2 & 100.00 & 0 & 0.00 & 0 & 0.00 \\
\hline C2 & 5 & 55.56 & 2 & 22.22 & 2 & 22.22 \\
\hline
\end{tabular}

Table 3 shows functional outcome based on type of fracture. It was observed that, all types of fractures had excellent outcome except type $\mathrm{C} 1$ and $\mathrm{C} 2$ where $55.56 \%$ and $66.66 \%$ of patients had excellent outcome respectively

\section{Discussion}

Distal third femur fractures is seen to be closely interconnected with high-energy injury (young) and osteoporosis (elders). Metaphyseal comminution has proven to be challenging in conventional plate fixation. The locking compression plate (LCP) is fixed angle construct with combination holes i.e compression as well as locking holes and thus provides the surgeon a chance to use any of them depending on individual fracture morphology.

It has been found that, an estimated $6 \%$ of all the femur fractures occur in the distal part of the bone..$^{18,19}$ The fractures occur with a bimodal distribution. One group includes patients below 40 years of age, predominantly males, sustaining high-energy trauma such as that in a traffic accident or a fall from height. The other group is comprising of patients $>50$ years, predominantly osteoporotic females, sustain low energy injury. 85 In both the cases, axial load to the leg is found to be the most common mechanism of injury. Less commonly rotation forces lead to distal femur fractures. ${ }^{19}$ About $60 \%$ of distal femoral fractures occur in the age group of $>50$. The associated osteoporosis found within this group may pose problems for fixation. ${ }^{18}$ Also seen are associated meniscal or ligamentous damage following distal femoral fractures, while the incidence of any neurovascular damage remains rare. ${ }^{20}$

In the present study the clinical and radiological union were noted to be present in $46.67 \%$ of patients each at an interval of three months and six months. Tenderness as a complication was present in $50 \%$ of patients at one and half month follow up while $10 \%$ of patients had tenderness at third and sixth month. Stiffness was present in $20 \%$ of patients while the complication of infection and pain were noted in $26.67 \%$ of patients each at first, second and third follow ups. The mean flexion achieved at 1.5 months follow up was $75.75 \pm 13.50$ which improved to $85.75 \pm 12.28$ and at six months follow up was $101.25 \pm 15.80$. The mean extension of the patients through the follow up period was found to be $1.75 \pm 3.73$. Using Sanders 40 point Functional evaluation scale, $80 \%$ of patients had excellent functional outcome and good and fair outcome was noted in $10 \%$ of patients respectively. The given outcome was comparable in both the sexes. Out of the 24 patients who had excellent functional outcome $15(71.43 \%)$ were males while all the nine females (100\%) had excellent outcome. However this difference was not significant statistically $(\mathrm{p}=0.2005)$.

Locking plate has a fixed angle between plate and screw interface. It eliminates screw toggle and reduces motion at fracture site, preserving the periosteal blood supply. It also provides a stronger fixation in cases of osteoporotic bone. Therefore it is an ideal implant for osteoporotic bone

The DF-LCP, an advancement of the LISS, was introduced in mid-late 1990 's. ${ }^{22,23}$ The primary distinction being that LISS uses an outrigger device for proximal holes, acting like locking guide jig, attached to distal part of plate which guides the placement of proximal locking screws. Shaft holes on DF-LCP are combination holes that allows both compression screw or locking screw for more precise positioning of plate.

The studies reveal that early functional results are analogous to final long term results, though the period of study is short. ${ }^{24}$ The results are associated with severity of fractures, anatomical reducibility, etiology, osteoporosis, time interval from trauma to surgery, associated injury, comorbidities and accurate implant 
placement and fixation. ${ }^{25}$

The long term prognosis stands uncertain, since the earliest LISS was used in the mid- late 1990's. Also, the initial associated damage to cartilage can result in early osteoarthritis even though there is no evidence of that yet. ${ }^{26}$ Previous studies have noted better outcomes in $\mathrm{A} 1$ and $\mathrm{C} 1$ types. ${ }^{24}$

\section{Conclusion}

In this study of using locking compression plate in the treatment of distal femur fractures based on Sanders 40 point Functional evaluation scale, excellent functional outcome was noted in $80 \%$ of patients and good and fair outcome was noted in $10 \%$ of patients each. This outcome was comparable in both males and females and in all the involved age groups. Further, excellent outcome was noted in all types of fractures except type $\mathrm{C} 1$ and $\mathrm{C} 2$ where almost two third of the patients had an excellent outcome.

Tenderness as a complication was noted in about half of the study population at one and half month follow up. However, it reduced during three and six months follow up. Stiffness as a complication was present in $20 \%$ while infection and pain were noted in $26.67 \%$ of patients each at first, second and third follow ups.

1. The DF-LCP should be implant of choice in among the present generation of implants available for fixation of fractures of the distal femur.

2. However, precision in positioning and fixation are required to produce satisfactory results.

3. We recommend DF-LCP implant in Type C and osteoporotic fractures.

\section{References}

1. Rockwood and Green's Fractures in adults. Robert Bucholz, James Heckman, editors. Lippincott Williams and Wilkins. $8^{\text {th }}$ edition. 2015;p2229-2230.

2. Crist BD, Della Rocca GJ, Murtha YM. Treatment of acute distal femur fractures. Orthopedics. 2008;31(7):681-90.

3. Wilson JN. Watson Jone's: Fractures and joint injuries. $6^{\text {th }}$ ed, pg.1003-070, 1982.

4. Charnley John. The closed treatment of common fractures. $3^{\text {rd }}$ ed, Pg.197-204.

5. Butt MS, Krikler SJ, Ali MS. Displaced fractures of the distal femur in elderly patients. Operative versus nonoperative treatment. J Bone Joint Surg Br. 1996; 78(1):110-114.

6. Lannacone WM, Taffet R, DeLong WG, Jr., Born CT, Dalsey RM, Deutsch LS. Early exchange intramedullary nailing of distal femoral fractures with vascular injury initially stabilized with external fixation. J Trauma. 1994;37(3):446-451.

7. Johnson KD HG. Distal femoral fractures. Orthop Clin North Am. 1987;18(1):115-132.

8. Ronen GM, Michaelson M, Waisbrod H. External fixation in war injuries. Injury. 1974; 6(2):94-98.

9. Distal Femoral Locking Compression Plate Fixation in Distal Femoral Fractures: Early Results.

10. Kregor PJ, Stannard J, Zlowodzki M, Cole PA, Alonso J. Distal femoral fracture fixation utilizing the Less Invasive Stabilization System (L.I.S.S.): The technique and early results. Injury 2001;32:SC32-47.

11. Schutz M, Muller M, Krettek C, et al. Minimally invasive fracture stabilization of distal femoral fractures with the LISS: A prospective multicenter study. Results of a clinical study with special emphasis on difficult cases. Injury 2001;3 2:SC 48-54.

12. Schutz M, Muller M, Regazzoni P, et al. Use of the Less Invasive Stabilization System (LISS) in patients with distal femoral (AO33) fractures: a prospective multicenter study. Arch Orthop Trauma Surg 2005; 125(2):102-8.

13. Schandelmaier P, Partenheimer A, Koenemann B, Grun OA, Krettek C. Distal Femoral Fractures and LISS Stabilization. Injury 2001;32:SC55-63.

14. Frigg R, Appenzeller A, Christensen R, Frenk A, Gilbert S, Schavan R. The development of the distal femur Less Invasive Stabilization System (LISS). Injury 2001;32:SC24-31.

15. Button G, Wolinsky P, Hak D. Failure of Less Invasive Stabilization System Plates in the Distal Femur:A Report of Four Cases. J Orthop Trauma 2004; 18(8): 565-70.

16. Yuvarajan $p$, rahul kaul, Lalit Maini, maulana aazad medical college, Delhi Review of concepts in distalfemoral fracture management. Pb Journal Of Orthopaedics.2009, Vol-11,pno.1.

17. John Scolaro, Jamino Ahn, University Of Pensylvania. Locked plating in practice, indications and current concepts. University of Pennsylvania Orthopaedic Journal. May 2011, Vol-21, p-18-21.

18. KOLMERT, L., WULFF, K.: Epidemiology and treatment of distal femoral fractures in adults. Acta Orthop. Scand., 53:957-962, 1982.

19. MARTINET, O., CORDEY J., HARDER, Y., MAIER, A., BÜHLER, M., BARRAUD, G. E.: The epidemiology of fractures of the distal femur. Injury, 31(Suppl 3):C62-63, 2000.

20. Link BC, Babst R. Current Concepts in Fractures of the Distal Femur. Acta Chirurgiae OrthopaedicaeEt Traumatologiae Čechosl 2012;79:11-20.

21. Kregor PJ, Stannard JA, Zlowodzki M, Cole PA. Treatment of distal femur fractures using the Less Invasive Stabilization System: surgical experience and early clinical results in 103 fractures. J Orthop Trauma 2004;18(8):50920.

22. Schandelmaier P, Partenheimer A, Koenemann B, Grun OA, Krettek C. Distal Femoral Fractures and LISS Stabilization. Injury 2001;32:SC 55-63.

23. Frigg R, Appenzeller A, Christensen R, Frenk A, Gilbert S, Schavan R. The development of the distal femur Less Invasive Stabilization System (LISS). Injury 2001;32:SC 24-31.

24. Bolhofner BR, Carmen B, Clifford P. The results of open reduction and internal fixation of distal femur fractures using a biologic (Indirect) Reduction Technique. J Orthop Trauma 1996;10(6):372-7.

25. Fankhauser F, Gruber G, Schippinger G, et al. minimalinvasive treatment of distal femoral fractures with the LISS (Less Invasive Stabilization System): A Prospective study of 30 fractures with a follow up of 20 months. Acta Orthop Scand 2004;75(1):56-60.

26. Markmiller M, Konrad G, Sudkamp N. Femur-LISS and distal femoral nail for fixation of distal femoral fractures. Clin Orthop 2004;426:252-7. 